\title{
Two numerical methods based on discretization of the MPS method for solving the two-dimensional Burgers' equation
}

\author{
H. Sakurai \\ Co-operative Education Center, Sendai National College of Technology, \\ Japan
}

\begin{abstract}
The Burgers' equation is an important and basic nonlinear partial differential equation in fluid dynamics, and has been used as a model equation in other fields, such as modeling of shock waves, gas dynamics, turbulence, and large bubble structures consisting of clusters of galaxies in space. Many researchers have proposed various numerical methods for solving the Burgers' equation, such as the finite difference method, finite element method, boundary element method, etc. The objective of the present research is to propose Cole-Hopf the transformation method (CHTM) and direct method (DM) based on discretization of the moving particle semi-implicit (MPS) method for solving the twodimensional Burgers' equation. The numerical results of one and two-dimensional problems are compared with exact solutions and other numerical solutions, and the validity of the present methods is shown.

Keywords: Burgers' equation, Cole-Hopf transformation, direct method, discretization of MPS method, two-dimensional problem.
\end{abstract}

\section{Introduction}

The Burgers' equation is an important and basic nonlinear partial differential equation in fluid dynamics, and has been used as a model equation in other fields, such as modeling of shock waves, gas dynamics, and turbulence, etc. [16]. It is well known that even if a smoothed initial condition is given, a shock wave having sharp serrated shape is formed in finite time. Furthermore, one characteristic of the nonlinear one-dimensional Burgers' equation is that it can be reduced to a linear one-dimensional diffusion equation using the Cole-Hopf 
transformation [5]. So far, many different numerical methods to solve the Burgers' equation, such as finite difference method [6-10], finite element method [11-13], boundary element method and meshless method [14-17] and others $[18,19]$ have been proposed.

On the other hand, in recent years, the Moving- Particle Semi-implicit (MPS) method has been attracting much interest [20, 21]. The MPS method is a leading numerical-analysis technique that has been used to investigate, complicated phenomena such as incompressible inviscid flows with free surfaces and the collapse of a column of water. One advantage of the MPS method is that it needs no integral calculations, unlike finite elements methods, and it is expected to greatly reduce CPU time. It is also a powerful method for phenomenon in which discontinuous points appear. However, to the best of the author's knowledge, no numerical solutions for the Burgers' equation by the MPS method have been reported.

The objective of the present research is to propose numerical methods based on the discretization of the MPS method for solving the two-dimensional Burgers' equations. When we can't obtain the solution of the partial differential equation of the Cole-Hopf transformation explicitly, we have to solve it numerically in advance to obtain the initial conditions for after the transformations. Further, we need extra calculations to carry out the inverse transformation to real space. This leads to increased CPU time. Therefore, another purpose of the present paper is to propose a numerical method without the Cole-Hopf transformation. In this paper, a numerical method which uses the Cole-Hopf transformation is called the "Cole-Hopf transformation method" (CHTM), and a numerical method without using the Cole-Hopf transformation is called the "direct method" (DM).

Several one and two-dimensional analysis are carried out by the two numerical methods. As a result, the solutions of the two methods show good agreement with the analytical solutions and other numerical solutions for one and two-dimensional problems, indicating the validities of the present methods.

\section{Governing equations}

In the Cartesian co-ordinate system $O-x y$, we consider the following system of two-dimensional nonlinear Burgers' equations :

$$
\begin{aligned}
& \frac{\partial u}{\partial t}+u \frac{\partial u}{\partial x}+v \frac{\partial u}{\partial y}=\frac{1}{R_{e}}\left(\frac{\partial^{2} u}{\partial x^{2}}+\frac{\partial^{2} u}{\partial y^{2}}\right),(x, y, t) \in D \times(0, T] \\
& \frac{\partial v}{\partial t}+u \frac{\partial v}{\partial x}+v \frac{\partial v}{\partial y}=\frac{1}{R_{e}}\left(\frac{\partial^{2} v}{\partial x^{2}}+\frac{\partial^{2} v}{\partial y^{2}}\right),(x, y, t) \in D \times(0, T]
\end{aligned}
$$

subject to the initial conditions:

$$
\begin{aligned}
& u(x, y, 0)=u_{0}(x, y),(x, y) \in D \\
& v(x, y, 0)=v_{0}(x, y),(x, y) \in D
\end{aligned}
$$

the boundary conditions: 


$$
\begin{aligned}
& u(x, y, t)=f(x, y, t),(x, y, t) \in \partial D \times(0, T] \\
& v(x, y, t)=g(x, y, t),(x, y, t) \in \partial D \times(0, T]
\end{aligned}
$$

and the potential symmetry condition:

$$
\frac{\partial u}{\partial y}=\frac{\partial v}{\partial x}
$$

where $D$ is a domain and $\partial D$ is its boundary. In the above equations, $t$ is time, and $u(x, y, t)$ and $\mathrm{v}(x, y, t)$ are the velocity components to be determined in the $x$ direction and the $y$ direction respectively, and $T$ is time of analysis. In addition, $u_{0}(x, y), v_{0}(x, y), f(x, y, t)$ and $g(x, y, t)$ are known functions and $R_{e}$ is the Reynolds number.

\section{Discretization of MPS method}

In this section, the discretization of the MPS method will be described briefly $[20,21]$. The discretization of the gradient vector of a scalar function $\phi(x, y)$ at the $i$-particle is given by eqn. (3.1):

$$
\langle\nabla \phi\rangle_{i}=\frac{d}{n_{i}} \sum_{j \neq i}\left[\frac{\phi_{j}-\phi_{i}}{\left|\boldsymbol{r}_{j}-\boldsymbol{r}_{i}\right|^{2}}\left(\boldsymbol{r}_{j}-\boldsymbol{r}_{i}\right) w\left(\boldsymbol{r}_{j}-\boldsymbol{r}_{i}\right)\right],
$$

where $\phi_{i}, \phi_{j}$ are the values of the scalar function $\phi(x, y), \boldsymbol{r}_{i}, \boldsymbol{r}_{j}$ are the position vectors of $i$-particle and $j$-particle respectively, $\left|\boldsymbol{r}_{i}-\boldsymbol{r}_{j}\right|$ is the distance between the $i$-particle and $j$-particle, $n_{i}$ is the particle density, $d$ is the dimensional number, the function $w$ is the weighted function and $\Sigma$ denotes summation with respect to $j \neq i$. The weighted function $w$ is given by the following eqn. (3.2):

$$
w(r)=\left\{\begin{array}{lr}
\frac{r_{e}}{r}-1 & \left(0 \leq r<r_{e}\right) \\
0 & \left(r_{e} \leq r\right)
\end{array},\right.
$$

where, $r$ is the distance between two particles, and $r_{e}$ is the radius of the interaction. Hence, if $r$ is less than $r_{e}$, there is interaction between two particles. The particle density $n_{i}$ is given by eqn.(3.3),

$$
n_{i}=\sum_{j \neq i} w\left(\boldsymbol{r}_{j}-\boldsymbol{r}_{i}\right)
$$

The discretization of the Laplacian operator of the scalar function $\phi(x, y)$ at the $i$-particle is given by eqn.(3.4):

$$
\left\langle\nabla^{2} \phi\right\rangle_{i}=\frac{2 d}{\lambda n_{i}} \sum_{j \neq i}\left[\left(\phi_{j}-\phi_{i}\right) w\left(\boldsymbol{r}_{j}-\boldsymbol{r}_{i}\right)\right],
$$

where, $\lambda$ is a constant given by the following eqn. (3.5): 


$$
\lambda=\frac{\sum_{j \neq i}\left|\boldsymbol{r}_{j}-\boldsymbol{r}_{i}\right|^{2} w\left(\left|\boldsymbol{r}_{i}-\boldsymbol{r}_{j}\right|\right)}{\sum_{j \neq i} w\left(\left|\boldsymbol{r}_{i}-\boldsymbol{r}_{j}\right|\right)} .
$$

\section{Analytical theory and basic equations for CHTM}

The well known Cole-Hopf transformation equations are given as follows [5]:

$$
\begin{gathered}
u(x, y, t)=-2 \frac{1}{R_{e}} \frac{1}{\phi} \frac{\partial \phi}{\partial x} \\
v(x, y, t)=-2 \frac{1}{R_{e}} \frac{1}{\phi} \frac{\partial \phi}{\partial y} .
\end{gathered}
$$

For instance, according to references [5, 13], we omit some details due to limitations of space though, the two-dimensional Burgers' eqns (2.1)-(2.2) can be transformed to the following linear diffusion eqn. (4.3) using the Cole-Hopf transformation:

$$
\frac{\partial \phi}{\partial t}=\frac{1}{R_{e}}\left(\frac{\partial^{2} \phi}{\partial x^{2}}+\frac{\partial^{2} \phi}{\partial y^{2}}\right)
$$

Making use of the Euler's explicit scheme to $\partial \phi / \partial t$, and applying the discretization of the MPS method to the Laplacian, we can derive the following basic eqn. (4.4) for the CHTM:

$$
\phi_{i}^{S+1}=\phi_{i}^{S}+\Delta t \cdot \frac{1}{R_{e}} \frac{2 d}{\lambda n_{i}} \sum_{j \neq i}\left[\left(\phi_{j}^{S}-\phi_{i}^{S}\right) w\left(\left|\boldsymbol{r}_{j}-\boldsymbol{r}_{i}\right|\right)\right]
$$

where the upper suffix $s$ denotes a time step number, the lower suffix $i$ means a particle number, and $\Delta t$ is a time increment. In the present study, we adopt the next forward finite difference approximations (4.5)-(4.6) to evaluate $\partial \phi / \partial x, \partial \phi / \partial x$ in eqns (4.1)-(4.2):

$$
\begin{aligned}
& \frac{\partial \phi}{\partial x}=\frac{\phi(x+d x, y, t)-\phi(x, y, t)}{d x} \\
& \frac{\partial \phi}{\partial y}=\frac{\phi(x, y+d y, t)-\phi(x, y, t)}{d y} .
\end{aligned}
$$

Therefore, in addition to calculation of the values $\phi(x, y, t)$, calculations of the values $\phi(x+d x, y, t)$ and $\phi(x, y+d y, t)$ are needed in the CHTM.

\section{Analytical theory and basic equations for DM}

In eqns (2.1)-(2.2), applying the Euler's explicit method to the time derivatives of the left hand side, and using the discretizations of the MPS method to the gradients and Laplacian, we can obtain the following basic eqns (5.1)-(5.2) for the DM: 


$$
\begin{gathered}
-\Delta t\left[\begin{array}{c}
u_{i}^{s} \cdot\left\{\frac{d}{n_{i}} \sum_{j \neq i} \frac{u_{j}^{s}-u_{i}^{s}}{\left|\boldsymbol{r}_{j}-\boldsymbol{r}_{i}\right|^{2}}\left(\boldsymbol{r}_{j}-\boldsymbol{r}_{i}\right) w\left(\left|\boldsymbol{r}_{j}-\boldsymbol{r}_{i}\right|\right)\right\}_{x} \\
+v_{i}^{s} \cdot\left\{\frac{d}{n_{i}} \sum_{j \neq i} \frac{u_{j}^{s}-u_{i}^{s}}{\left|\boldsymbol{r}_{j}-\boldsymbol{r}_{i}\right|^{2}}\left(\boldsymbol{r}_{j}-\boldsymbol{r}_{i}\right) w\left(\left|\boldsymbol{r}_{j}-\boldsymbol{r}_{i}\right|\right)\right\}_{y}
\end{array}\right] \\
+\Delta t \cdot \frac{1}{R_{e}} \cdot \frac{d}{\lambda n_{i}} \sum_{j \neq i}\left[\left(u_{j}^{s}-u_{i}^{s}\right) w\left(\left|\boldsymbol{r}_{j}-\boldsymbol{r}_{i}\right|\right)\right] \\
v_{i}^{s+1}=v_{i}^{s} \cdot \Delta t \quad\left[\begin{array}{l}
u_{i}^{s} \cdot\left\{\frac{d}{n_{i}} \sum_{j \neq i} \frac{v_{j}^{s}-v_{i}^{s}}{\left|\boldsymbol{r}_{j}-\boldsymbol{r}_{i}\right|^{2}}\left(\boldsymbol{r}_{j}-\boldsymbol{r}_{i}\right) w\left(\left|\boldsymbol{r}_{j}-\boldsymbol{r}_{i}\right|\right)\right\}_{x} \\
-\Delta t \\
+v_{i}^{s} \cdot\left\{\frac{d}{n_{i}} \sum_{j \neq i} \frac{v_{j}^{s}-v_{i}^{s}}{\left|\boldsymbol{r}_{j}-\boldsymbol{r}_{i}\right|^{2}}\left(\boldsymbol{r}_{j}-\boldsymbol{r}_{i}\right) w\left(\left|\boldsymbol{r}_{j}-\boldsymbol{r}_{i}\right|\right)\right\}_{y}
\end{array}\right] \\
+\Delta t \cdot \frac{1}{R_{e}} \cdot \frac{d}{\lambda n_{i}} \sum_{j \neq i}\left[\left(v_{j}^{s}-v_{i}^{s}\right) w\left(\left|\boldsymbol{r}_{j}-\boldsymbol{r}_{i}\right|\right)\right] .
\end{gathered}
$$

In eqns (5.1)-(5.2), the upper suffix $s$ of the velocity components $u_{i}^{s}$ and $v_{i}^{s}$ indicates a time step number, and the terms \{\}$_{x}$ and \{\}$_{y}$ in the right hand side, denote the $x$ and $y$ components of the gradient vectors $\nabla u$ and $\nabla v$.

\section{Test problems and discussion}

In the following analysis, the radius of the interaction $r_{e}$ in eqn.(3.2) is set to two times of the minimum distance between two particles. Values from two to four times of the minimum distance between two arbitrary particles is recommended in references [20, 21].

\subsection{One-dimensional problem (1)}

We consider the following initial condition and boundary condition:

$$
\begin{gathered}
u(x, 0)=\sin (\pi x), \quad 0<x<1 \\
u(0, t)=u(1, t)=0 \quad 0 \leq t .
\end{gathered}
$$

In both the CHTM and the DM, the numerical computations were performed using uniform 41 particles, the time increment $\Delta t=1.0 \times 10^{-3}$, and $d x=$ $1.0 \times 10^{-6}$ in the forward difference approximation eqn. (4.5) of the Cole-Hopf transformation.

Figures 1 and 2 show the results of the CHTM and the DM with exact solutions for $R_{e}=100$ [9-11, 14]. These figures illustrate the velocity of the fluid at time $t=0.2,0.4,0.6,0.8,1.0$. Comparing the results of the CHTM and the DM, we can see that the profiles of the CHTM are a little bit sharper than those of the DM near the right-hand boundary. 


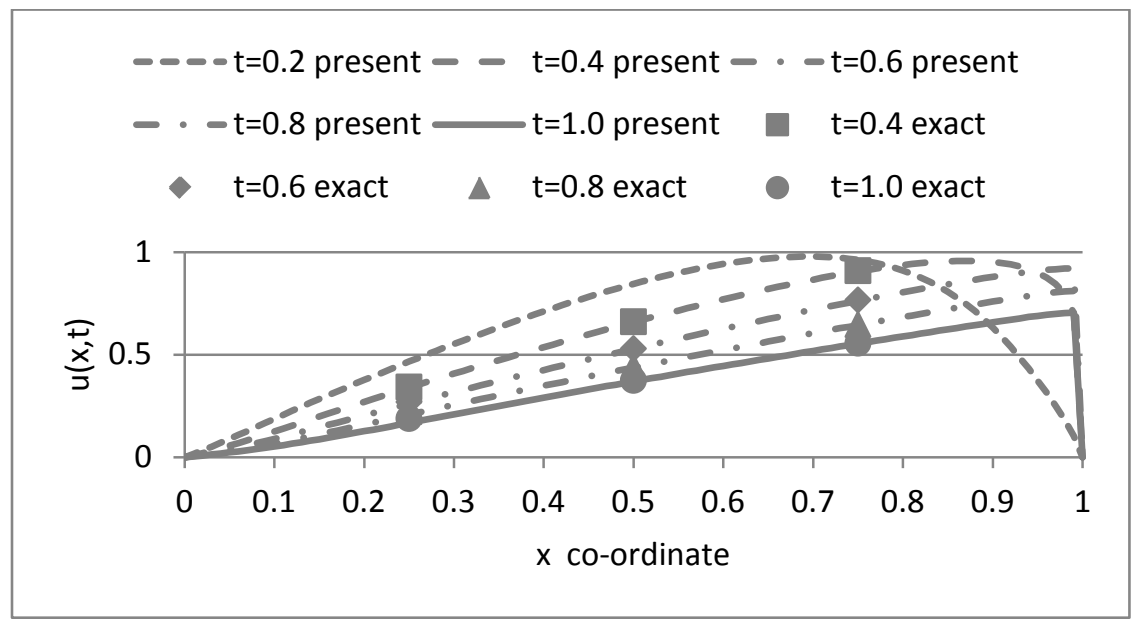

Figure 1: Numerical solutions of one-dimensional problem(1) by the CHTM at different times for $R_{e}=100, \Delta t=1.0 \times 10^{-3}$.

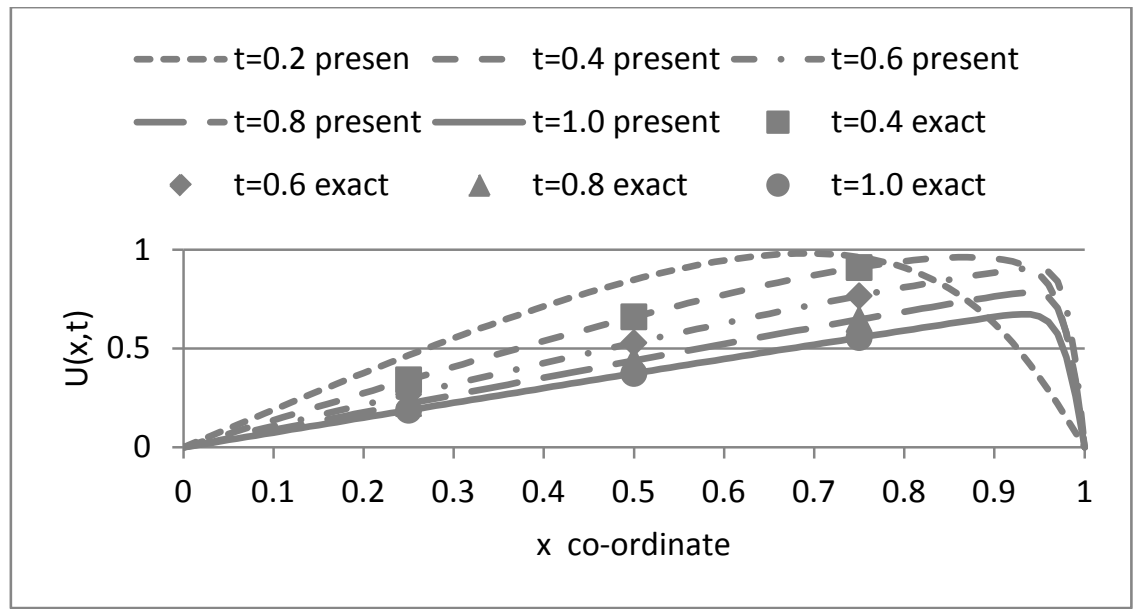

Figure 2: $\quad$ Numerical solutions of one-dimensional problem (1) by the DM at different times for $R_{e}=100, \Delta t=1.0 \times 10^{-3}$.

\subsection{One-dimensional problem (2)}

Now, as the second verification problem, we will consider a one-dimensional problem treated in reference [7].

The initial condition and the boundary condition of the problem are given as follows respectively: 


$$
\begin{array}{cl}
u(x, 0)=\frac{2 \pi \sin \pi x+8 \pi \sin 2 \pi x}{4+\cos \pi x+2 \cos 2 \pi x}, & 0<x<1 \\
u(0, t)=u(1, t)=0 & 0 \leq t .
\end{array}
$$

The numerical computation was performed using uniform 41 particles along the $x$ axis and the time increment $\Delta t=1.0 \times 10^{-4}$.

The results by the DM and the exact solutions at different time levels for $R_{e}=1.0$ are shown in Figure 3. In reference [7], the equation of the exact solution of the present problem has been given as follows:

$$
u(x, t)=\frac{2 \pi\left[\exp \left(-\pi^{2} t\right) \sin \pi x+4 \exp \left(-4 \pi^{2} t\right) \sin 2 \pi x\right]}{4+\exp \left(-\pi^{2} t\right) \cos \pi x+2 \exp \left(-4 \pi^{2} t\right) \cos 2 \pi x} .
$$

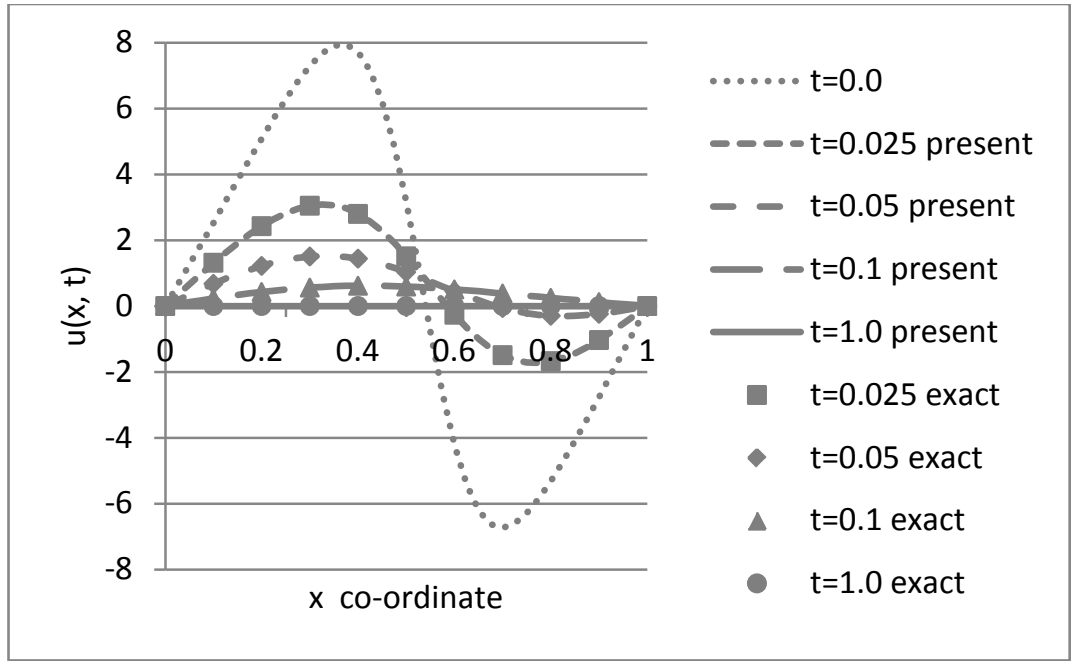

Figure 3: Numerical solutions of one-dimensional problem(2) by the DM at different times for $R_{e}=1.0, \Delta t=1.0 \times 10^{-4}$.

We confirmed that at the time step $t=1.0$, the relative maximum error between the present result and the exact solution is about zero percent again.

\subsection{Two-dimensional problem (1)}

We consider the Burger's eqns (2.1)-(2.2) over a square domain $D=$ $\{(x, y): 0 \leq x \leq 1,0 \leq y \leq 1\}$, with the initial conditions:

$$
\left.\begin{array}{l}
u(x, y, 0)=\sin (\pi x) \cos (\pi y) \\
v(x, y, 0)=\cos (\pi x) \sin (\pi y)
\end{array}\right\} \quad 0 \leq x \leq 1,0 \leq y \leq 1,
$$

and boundary conditions: 


$$
\left.\begin{array}{rl}
u(0, y, t) & =u(1, y, t)=0 \\
u(x, 0, t) & =u(x, 1, t)=0 \\
\partial u / \partial n(x, 0, t) & =\partial u / \partial n(x, 1, t)=0 \\
\partial v / \partial n(0, y, t) & =\partial v / \partial n(1, y, t)=0
\end{array}\right\} \quad 0 \leq t .
$$

The numerical computations were performed in the CHTM and the DM, using 21 particles in the $x$ and $y$ direction respectively, total 441 particles located uniformly, the time increment $\Delta t=1.0 \times 10^{-3}$, for the $R_{e}=100$. In addition, the value $d x=1.0 \times 10^{-3}$ is used in the forward difference approximation eqns (4.5)-(4.6) of the CHTM. The results analyzed by the CHTM and the DM at different times are shown in Figures 4 and 5 respectively. The results in Figures 4 and 5 denote the distributions of $u(x, x, t)$ along the diagonal line of the square region, and EFCGM means results of the element-free characteristic Galerkin method [17]. And, by antisymmetry of the results, the present results are plotted in the range for only $0 \leq x \leq 0.5$. We can see that the results of both methods are in good agreement with those of the EFCGM. Through the present analysis, the solutions by the DM can be considered to have good accuracy as approximations again.

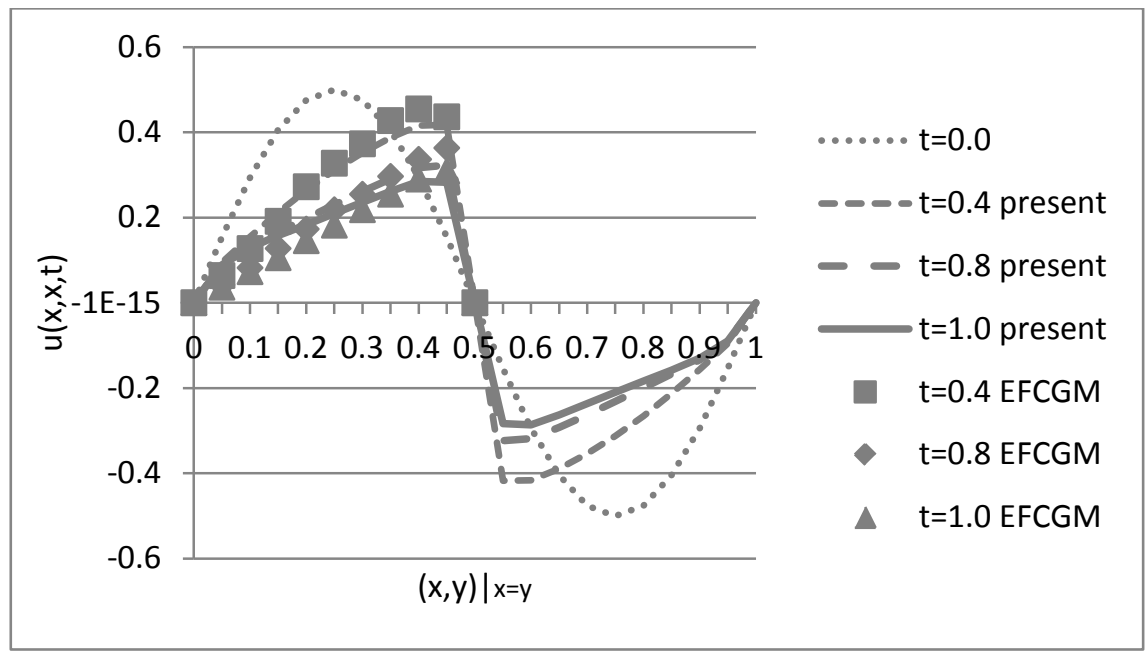

Figure 4: Numerical solutions of two-dimensional problem(1) by the CHTM at different times for $R_{e}=100, \Delta t=1.0 \times 10^{-3}$. 


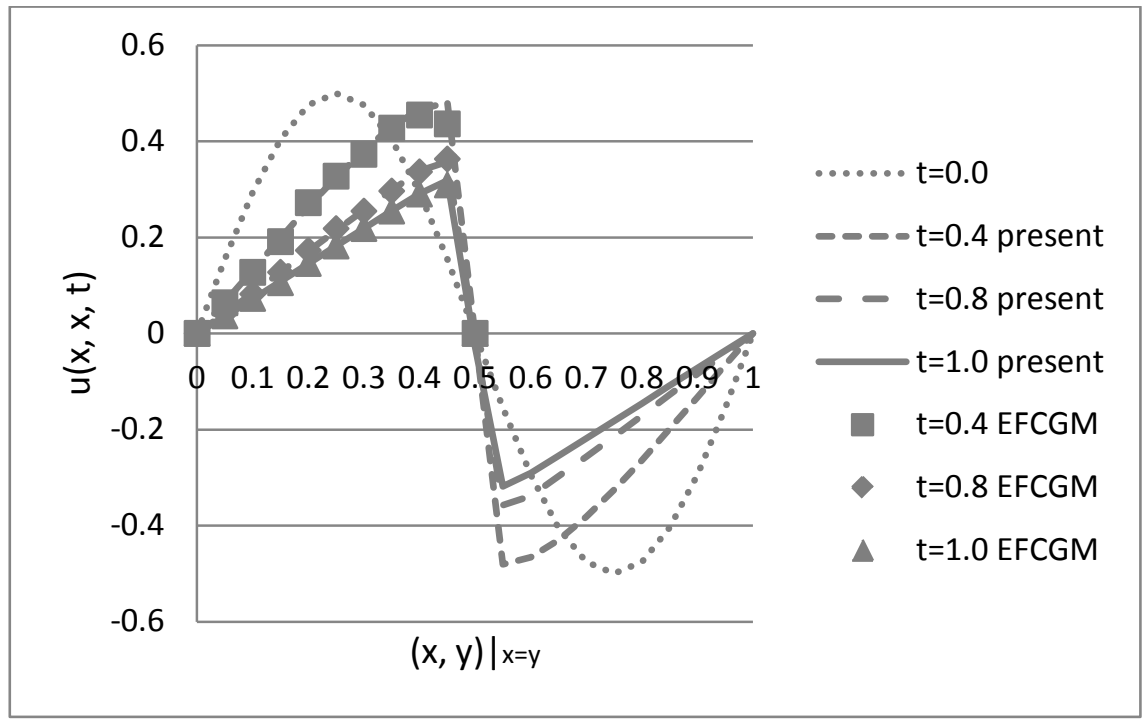

Figure 5: Numerical solutions of two-dimensional problem (1) by the DM at different times for $R_{e}=100, \Delta t=1.0 \times 10^{-3}$.

\subsection{Two-dimensional problem (2)}

Let us consider the other two-dimensional problems [6, 8, 17]. The exact solution of the two-dimensional eqns (2.1)-(2.2) can be generated by using the Cole-Hopf transformation which is:

$$
\begin{aligned}
& u(x, y, t)=\frac{3}{4}-\frac{1}{4\left[1+\exp \left((-4 x+4 y-t) \mathrm{R}_{\mathrm{e}} / 32\right)\right]}, \\
& v(x, y, t)=\frac{3}{4}+\frac{1}{4\left[1+\exp \left((-4 x+4 y-t) R_{e} / 32\right)\right]} .
\end{aligned}
$$

Table 1: $\quad$ Comparison of present DM with exact values of $\boldsymbol{u}$ for $\boldsymbol{R}_{\boldsymbol{e}}=\mathbf{1 0 0}$.

\begin{tabular}{|l|c|c|c|c|c|c|}
\cline { 2 - 7 } \multicolumn{1}{c|}{} & \multicolumn{2}{c|}{$\mathrm{t}=0.01$} & \multicolumn{2}{c|}{$\mathrm{t}=0.5$} & \multicolumn{2}{c|}{$\mathrm{t}=2.0$} \\
\hline coordinate & present & exact & present & exact & present & exact \\
\hline$(0.1,0.1)$ & 0.62243 & 0.62305 & 0.53346 & 0.54332 & 0.50036 & 0.50048 \\
\hline$(0.5,0.1)$ & 0.50161 & 0.50162 & 0.50025 & 0.50035 & 0.50000 & 0.50000 \\
\hline$(0.9,0.1)$ & 0.50001 & 0.50001 & 0.50000 & 0.50000 & 0.50000 & 0.50000 \\
\hline$(0.3,0.3)$ & 0.62315 & 0.62305 & 0.51551 & 0.54332 & 0.50017 & 0.50048 \\
\hline$(07,0.3)$ & 0.50161 & 0.50162 & 0.50006 & 0.50035 & 0.50000 & 0.50000 \\
\hline$(0.5,0.5)$ & 0.62315 & 0.62305 & 0.53422 & 0.54332 & 0.50010 & 0.50048 \\
\hline$(0.9,0.5)$ & 0.50161 & 0.50162 & 0.50023 & 0.50035 & 0.50000 & 0.50000 \\
\hline$(0.7,0.7)$ & 0.62315 & 0.62305 & 0.54147 & 0.54332 & 0.50025 & 0.50048 \\
\hline$(0.1,0.9)$ & 0.74999 & 0.74999 & 0.74989 & 0.74995 & 0.74004 & 0.74426 \\
\hline$(0.9,0.9)$ & 0.62305 & 0.62305 & 0.53796 & 0.54332 & 0.50041 & 0.50048 \\
\hline
\end{tabular}


Table 2: $\quad$ Comparison of present DM with exact values of $\boldsymbol{v}$ for $\boldsymbol{R}_{\boldsymbol{e}}=\mathbf{1 0 0}$.

\begin{tabular}{|l|c|c|c|c|c|c|}
\cline { 2 - 7 } \multicolumn{1}{c|}{} & \multicolumn{2}{c|}{$\mathrm{t}=0.01$} & \multicolumn{2}{c|}{$\mathrm{t}=0.5$} & \multicolumn{2}{c|}{$\mathrm{t}=2.0$} \\
\hline coordinate & present & exact & present & exact & present & exact \\
\hline$(0.1,0.1)$ & 0.87758 & 0.87695 & 0.96654 & 0.95668 & 0.99964 & 0.99952 \\
\hline$(0.5,0.1)$ & 0.99839 & 0.99838 & 0.99975 & 0.99965 & 1.00000 & 1.00000 \\
\hline$(0.9,0.1)$ & 0.99999 & 0.99999 & 0.99999 & 1.00000 & 1.00000 & 1.00000 \\
\hline$(0.3,0.3)$ & 0.87685 & 0.87695 & 0.98449 & 0.95668 & 0.99983 & 0.99952 \\
\hline$(07,0.3)$ & 0.99839 & 0.99838 & 0.99994 & 0.99965 & 1.00000 & 1.00000 \\
\hline$(0.5,0.5)$ & 0.87685 & 0.87695 & 0.96578 & 0.95668 & 0.99990 & 0.99952 \\
\hline$(0.9,0.5)$ & 0.99839 & 0.99838 & 0.99977 & 0.99965 & 1.00000 & 1.00000 \\
\hline$(0.7,0.7)$ & 0.87685 & 0.87695 & 0.95853 & 0.95668 & 0.99975 & 0.99952 \\
\hline$(0.1,0.9)$ & 0.75001 & 0.75001 & 0.75011 & 0.75005 & 0.75996 & 0.75574 \\
\hline$(0.9,0.9)$ & 0.87695 & 0.87695 & 0.96204 & 0.95668 & 0.99959 & 0.99952 \\
\hline
\end{tabular}

The computational domain is a square $D=\{(x, y): 0 \leq x \leq 1,0 \leq y \leq 1\}$, and the initial and boundary conditions are taken from the exact solution. The analysis was performed by the DM using the uniform 441 particles and the time increment $\Delta t=1.0 \times 10^{-3}$. Tables 1 and 2 give the numerical and exact values of $u(x, y, t)$ and $v(x, y, t)$ at some typical particle points for $R_{e}=100$ at time steps $t=0.01,0.5,2.0$. The solutions by the DM can be considered to have good accuracy as an approximation again.

\section{Conclusions}

In this paper, two numerical methods based on the discretization of the MPS method for solving the two-dimensional nonlinear Burgers' equation have been proposed.

Carrying out the several analyses of the one-dimensional and twodimensional problems, we can see that numerical results obtained by the both proposed methods are in good agreement with the exact solutions and the other numerical solutions. Therefore, the accuracy of the numerical solutions indicates that both the CHTM and the DM methods are well suited to solve the Burgers' equations. Especially, the accuracy of the result by the DM was also shown.

However, as a rule, it is difficult to claim predominance of the CHTM and DM. It is important to select a good method according to initial conditions, boundary conditions, size of the degree of the freedom, and shapes of analysis domain etc.

Future work will be focused on analysis of large scale problems and practical problems in fields such as aerospace.

\section{References}

[1] J. M. Burgers, A mathematical model illustrating the theory of turbulence, Adv. Appl. Mech. I (1948), 171-199.

[2] Julian D. Cole, On a quasi-linear parabolic equation occurring in aerodynamics, Quarterly of Applied Mathematics, No.3 (1951), 225-236. 
[3] E. Hopf, The partial differential equation $u_{t}+u u_{x}=\mu_{x x}$, Commun. Pure Appl. Math., 3(1950), 201-30.

[4] P. C. Jain and D. N. Holla, Numerical Solutions of Coupled Burgers' Equation, International Journal of Non-Linear Mechanics, Vol.13 (1978), 213-222.

[5] Clive A. J. Fletcher, General Exact Solutions of the Two-Dimensional Burgers’ Equations, International Journal for Numerical Methods in Fluid, Vol.3 (1983), 213-216.

[6] Wenyuan Liao, A fourth-order finite-difference method for solving the system of two-dimensional Burgers' equations, International Journal for Numerical Methods in Fluid, 64 (2010), 65-590.

[7] Tony W. H. Sheu and C. F. Chen and L. W. Hsieh, Development of a sixthorder two-dimensional convection-diffusion scheme via Cole-Hopf transformation, Computers Methods in Applied Mechanics and Engineering, 191 (2002), 2979-2995.

[8] Refik Bahadir, A fully implicit finite-difference scheme for twodimensional Burgers' equations, Applied Mathematics and Computation, 137 (2003), 131-137.

[9] Mohan. K. Kadalbajoo and A. Awasthi, A numerical method based on Crank-Nicolson scheme for Burgers' equation, Applied Mathematics and Computation, 182 (2006), 1430-1442.

[10] K. Pandey, Lajja Verma and Amit K. Verma, On a finite difference scheme for Burgers’ equation, Applied Mathematics and Computation, 215 (2009), 2206-2214.

[11] T. Ozis, E. N. Aksan and A. Ozdes, A finite element approach for solution of Burgers' equation, Applied Mathematics and Computation, 139 (2003), 417-428.

[12] E. N. Aksan, A numerical solution of Burgers' equation by finite element method constructed on the method of discretization in time, Applied Mathematics and Computation, 170 (2005), 895-904.

[13] Guozhong Zhao, Xijun Yu and Rongpei Zhnag, The new numerical method for solving the system of two-dimensional Burgers' equations, Computers and Mathematics with Applications, 62 (2011), 3279-3291.

[14] Refik Bahadir, Mustafa Saglam, A mixed finite difference and boundary element approach to one-dimensional Burgers' equation, Applied Mathematics and Computation, 160 (2005), 663-673.

[15] D. L. Young, C. M. Fan, S. P. Hu and S. N. Atluri, The EulerianLagrangian method of fundamental solutions for two-dimensional unsteady Burgers' equations, Engineering Analysis with Boundary Elements, 32 (2008), 395-412.

[16] Alireza Hashemian and Hossein M. Shodja, A meshless approach for solution of Burgers' equation, Journal of Computational and Applied Mathematics, 220 (2008), 226-239.

[17] Xiao Hua Zhang, Jie Ouyang, and Lin Zhang, Element-free characteristic Galerkin method for Burgers' equation, Engineering Analysis with Boundary Elements, 33 (2009), 356-362. 
[18] A. H. Khater, R. S. Temsah and M. M. Hassan, A Chebyshev spectral collocation method for solving Burgers'-type equations, Journal of Computational and Applied Mathematics, 222 (2008), 333-350.

[19] Fang Liu and Weiping Shi, Numerical solutions of two-dimensional Burgers' equations by lattice Boltzmann method, Commun Nonlinear Sci Numer Simulat, 16 (2011), 150-157.

[20] S. Koshizuka, A. Nobe and Y. Oka, Numerical Analysis of Breaking Waves using the Moving Particle Semi-Implicit Method, International Journal for Numerical Methods in Fluids, 26 (1998), 751-769.

[21] Ataie-Ashtiani, B. and Farhadi, L., A stable moving-implicit method for free surface flows, Fluid Dynamics Research 38 (2006), 241-256. 